\title{
Klinische Anwendungen von wassergefiltertem Infrarot A (wIRA) - eine Übersicht
}

\section{Clinical Applications of Water-Filtered Infrared-A (wIRA) - A Review}

(c) (i)

\author{
Autor \\ Gerd Hoffmann
}

Institut

Institut für Sportwissenschaften, Johann Wolfgang Goethe-

Universität, Frankfurt/Main

\section{Schlüsselwörter}

wassergefiltertes Infrarot A (wIRA), physikalische Medizin, Schmerzminderung, Gewebetemperatur, Wundheilung, Gewebe-Sauerstoffpartialdruck

\section{Key words}

water-filtered infrared-A (wIRA), physical medicine, reduction of pain, tissue temperature, wound healing, tissue oxygen partial pressure

eingereicht $\quad 19.02 .2017$

angenommen 06.06.2017

\section{Bibliografie}

DOI https://doi.org/10.1055/s-0043-113047

Phys Med Rehab Kuror 2017; 27: 265-274

(c) Georg Thieme Verlag KG Stuttgart · New York ISSN 0940-6689

Korrespondenzadresse

Prof. Dr. med. Gerd Hoffmann

Institut für Sportwissenschaften

Johann Wolfgang Goethe-Universität

Ginnheimer Landstraße 39

60487 Frankfurt/Main

Hoffmann@em.uni-frankfurt.de

\section{ZUSAMMENFASSUNG}

Wassergefiltertes Infrarot A (wIRA) ist eine spezielle Form der Wärmestrahlung mit hohem Eindringvermögen in das Gewebe und geringer thermischer Belastung der Hautoberfläche. wIRA steigert deutlich Temperatur, Sauerstoffpartialdruck und Durchblutung im Gewebe und wirkt auch über nicht-thermische zelluläre Effekte.
WIRA mindert indikationsübergreifend Schmerzen (mit relevant weniger Analgetikabedarf), Entzündung und vermehrte Sekretion und fördert Infektionsabwehr und Regeneration. Entsprechend breit sind die klinischen Anwendungsmöglichkeiten von wIRA.

wIRA ist ein kontaktfreies, verbrauchsmaterialfreies, leicht anzuwendendes, (selbst bei Wunden) als angenehm empfundenes Verfahren mit guter Tiefenwirkung und anhaltendem Wärmedepot.

wIRA ist u. a. einsetzbar zur Verbesserung der Heilung akuter und chronischer Wunden (wobei selbst eine ungestört "normal” ablaufende Wundheilung noch verbessert werden kann: schneller, schmerzärmer), bei Hauterkrankungen (wie vulgären Warzen, Herpes labialis, Herpes Zoster, Sklerodermie, Akne papulopustulosa; aktinischen Keratosen im Rahmen einer Photodynamischen Therapie), zur Resorptionsverbesserung topisch applizierter Substanzen, bei muskuloskeletalen Erkrankungen (wie Arthrosen, Arthritiden, Lumbago, ankylosierender Spondyloarthritis), zur Regeneration nach Sport, beim komplexen regionalen Schmerzsyndrom (CRPS), bei Polyneuropathien und in Kombination mit Strahlentherapie oder Chemotherapie in der Onkologie.

\section{ABSTRACT}

Water-filtered infrared-A (wIRA) is a special form of heat radiation with high tissue penetration and a low thermal load to the skin surface.

WIRA increases temperature, oxygen partial pressure and perfusion in the tissue markedly. wIRA has as well non-thermal cellular effects.

wIRA alleviates pain (with a substantially reduced need for analgesics), diminishes inflammation and increased exudation, and promotes infection defense and regeneration, all in a crossindication manner.

Indications for clinical applications of wIRA are accordingly wide.

wIRA is a non-contact, substance-free, easily usable, (even in wounds) pleasant feeling method with high tissue penetration and prolonged heat depot.

wIRA can be used i. a. for the promotion of healing of acute and chronic wounds (even an undisturbed "normal” wound healing 
can be improved: faster, with less pain), in skin diseases (e. g. common warts, Herpes simplex labialis, Herpes zoster, sclerodermia, acne papulopustulosa; actinic keratosis within a photodynamic therapy), for the resorption improvement of topically applied substances, in mu- sculoskeletal disorders (e.g. arthrosis, arthritis, spondyloarthritis), for regeneration after sports, in complex regional pain syndrome (CRPS), in polyneuropathies, and in combination with radiation therapy and chemotherapy in oncology.

\section{Grundlagen und Wirkungsweise}

Wassergefiltertes Infrarot A (wIRA) ist eine spezielle Form der Infrarotstrahlung (Wärmestrahlung) im Bereich von 780-1400 nm, die aufgrund ihrer sehr guten Verträglichkeit - im Gegensatz zur deutlich schlechteren Verträglichkeit von ungefiltertem Infrarot in der Medizin zu Prävention und Therapie verwendet wird [17]. So benutzen in Deutschland z. B. ca. 30\% der niedergelassenen Dermatologen und eine Reihe renommierter Kliniken, auch im operativen Bereich, wIRA.

Wassergefiltertes Infrarot A steigert deutlich Temperatur, Sauerstoffpartialdruck und Durchblutung im Gewebe [13, 16, 17, 23].

Diese 3 Faktoren sind entscheidend für die ausreichende Versorgung des Gewebes mit Energie und Sauerstoff. Hiervon hängen zahlreiche Leistungen des menschlichen Körpers ab, wie generell Regenerations- und Heilungsprozesse, z. B. Wundheilung und Infektionsabwehr [13, 16, 17, 36].

Wassergefiltertes Infrarot A mindert indikationsübergreifend Schmerzen, Entzündung und vermehrte Sekretion und verbessert Infektionsabwehr und Regeneration [13, 17, 23, 53].

Wassergefiltertes Infrarot A entspricht dem Großteil der in gemäßigten Klimazonen die Erdoberfläche wassergefiltert erreichenden Infrarotstrahlung der Sonne (Filterwirkung des Wassers und des Wasserdampfs der Erdatmosphäre). Durch die Wasserfilterung werden die Strahlungsanteile gemindert (die sogenannten Wasserabsorptionsbanden innerhalb des Infrarot $A$ und die meisten Teile des Infrarot B und C), die sonst durch Wechselwirkung mit Wassermolekülen in der Haut eine unerwünschte thermische Belastung (mit stechend-brennendem Gefühl) hervorrufen würden $[13,16,17]$. Technisch wird wIRA in speziellen Strahlern erzeugt (wIRA-Strahler), in denen die gesamte Strahlung eines Halogenstrahlers durch eine Wasser enthaltende Küvette hindurchtritt $[16,17,23,53]$. Während konventionelle Halogenstrahler ohne Wasserfilter („Rotlichtlampen“) je nach korrelierter Farbtemperatur 50-80\% ihrer Strahlung im unerwünschten Infrarot-B- und Infrarot-C-Bereich emittieren, ist dieser Anteil bei wIRA-Strahlern $<0,5 \%$, > Abb. $1[19,41,42]$.

Wassergefiltertes Infrarot A als spezielle Form der Infrarotstrahlung (Wärmestrahlung) mit hohem Eindringvermögen in das Gewebe bei geringer thermischer Oberflächenbelastung erlaubt einen deutlich höheren Energieeintrag in das Gewebe und wirkt sowohl über thermische (auf Wärmeenergietransfer bezogene) und temperaturabhängige (mit Temperaturänderung auftretende) als auch über nicht-thermische (ohne relevanten Wärmeenergietransfer) und temperaturunabhängige (ohne relevante Temperaturänderung auftretende) Effekte [13, 16, 17].

Innerhalb des Spektrums der Infrarot-A-Strahlung wurden Effekte von den energiereichen Wellenlängen nahe dem sichtbaren Licht - ungefähr 780-1000 nm, insbesondere 820 nm [27] - sowohl in vitro als auch in vivo beschrieben. Diese Wellenlängen stellen - v. a. im Hinblick auf nicht-thermische Effekte, wie die Minderung der Wundsekretion - den klinisch wichtigsten Teil von Infrarot A und wassergefiltertem Infrarot A dar [17]. Die Bestrahlungsstärken in diesem Wellenlängenbereich sind bei wIRA-Strahlern deutlich höher als bei konventionellen Halogenstrahlern ohne Wasserfilter: Bei einer Wellenlänge von $820 \mathrm{~nm}$ ist die bei gleicher Hautoberflächentemperatur anwendbare Bestrahlungsstärke bei einem wIRA-Strahler bspw. ca. 6-mal so hoch wie bei dem in der $\mathbf{A b b}$. 1 dargestellten Halogenstrahler ohne Wasserfilter mit einer korrelierten Farbtemperatur („,correlated color temperature“, CCT) von $3000 \mathrm{~K}$ und ca. 30-mal so hoch wie bei dem Halogenstrahler ohne Wasserfilter mit einer CCT von $1750 \mathrm{~K}[19,41,42]$.

Die Schmerzminderung durch wIRA wird sowohl über thermische als auch nicht-thermische Wirkungen erklärt [7, 8, 15, 17]: eine gesteigerte Durchblutung erlaubt eine bessere Elimination akkumulierter Metabolite, wie Schmerzmediatoren, Laktat und Bakterientoxine, und steigert zusammen mit der erhöhten Gewebetemperatur den Stoffwechsel (verbesserte Metabolisierung akkumulierter Stoffe und Regeneration); nicht-thermische Effekte beinhalten direkte Wirkungen auf Zellen und zelluläre Strukturen und Substanzen und möglicherweise auch auf Nozizeptoren. wIRA wirkt deutlich muskelentspannend und auch hierüber schmerzmindernd. Eine Schmerzminderung [40] bewirkt - neben einer Verbesserung der Lebensqualität - auch eine Erhöhung des Sauerstoffpartialdrucks $[1,7,8]$ und senkt damit deutlich das Infektionsrisiko [7, 8, 15, $17,25]$.

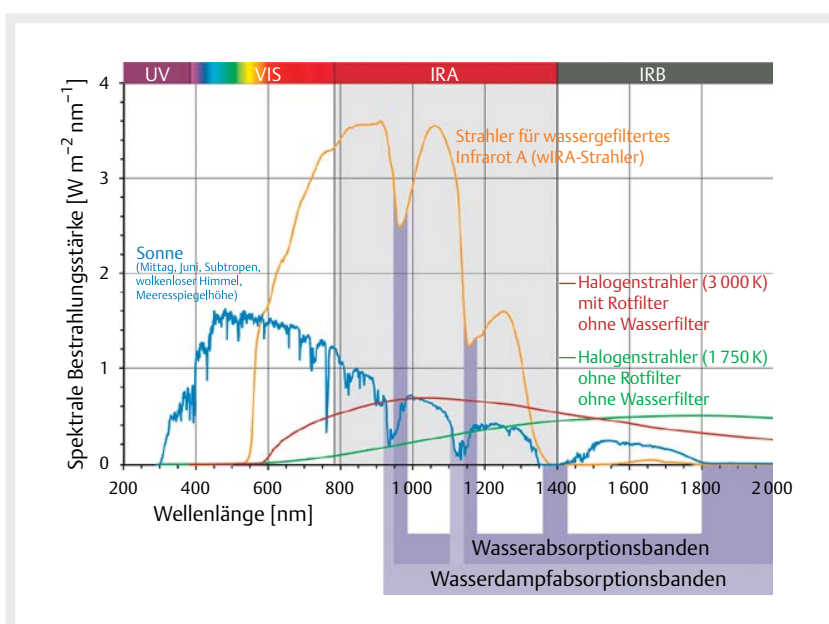

- Abb. 1 Spektren der Sonne, eines Strahlers für wassergefiltertes Infrarot A und von 2 Halogenstrahlern ohne Wasserfilter: Die 3 verschiedenen Strahler bewirken mit ihren dargestellten spektralen Bestrahlungsstärken die gleiche Hautoberflächentemperatur $[19,41,42,53]$. 
Außerdem wirkt eine Bestrahlung mit sichtbarem Licht („visible light“, VIS) und wIRA in Verbindung mit endogenem Protoporphyrin IX (oder Protoporphyrin IX von Bakterien) vermutlich gewissermaßen wie eine milde photodynamische Therapie (endogener PDTähnlicher Effekt) $[16,17,50]$. Dies fördert die Zellregeneration und Wundheilung (alte oder vorgeschädigte Zellen gehen in die Apoptose und werden durch neue Zellen ersetzt) und wirkt antibakteriell/antiinfektiv (photodynamische Inaktivierung von Bakterien) [17].

Einen wesentlichen Anteil an der antiinfektiven - antibakteriellen und antiviralen - Wirkung von wIRA dürfte die Verbesserung der körpereigenen Abwehr durch Steigerung von Temperatur, Sauerstoffpartialdruck und Durchblutung des Gewebes sowie der damit verbesserten Energie- und Sauerstoffbereitstellung für die körpereigene Abwehr haben, also ein thermischer wIRA-Effekt. Dieser ist vermutlich kombiniert mit nicht-thermischen wIRA-Effekten z. B. auf immunkompetente Zellen, sodass klinisch eine bessere Abwehrlage resultiert [17].

Die Wirkmechanismen von wIRA, z. B. über die Beeinflussung der Cytochrom-c-Oxidase, werden ausführlich in $[13,17]$ dargestellt.

\section{Große Anwendungsbreite}

wIRA sollte grundsätzlich immer dann in Erwägung gezogen werden, wenn klinisch eine tiefenwirksame Wärmeapplikation (mit hoher Leistungsdichtetoleranz und hohem Energiefluss ins Gewebe) erwünscht/indiziert ist oder wenn pathogenetisch mind. ein Faktor (Temperatur, Sauerstoffpartialdruck, Durchblutung im Gewebe) gestört oder suboptimal ist oder ein Symptom (z. B. Schmerz, Entzündung, Hypersekretion, Infektion, Regenerationsbedarf) vorliegt, das durch die thermischen und nicht-thermischen Effekte von wIRA positiv beeinflusst wird. Dabei werden selbst ungestört "normal" ablaufende Vorgänge, wie eine Regeneration nach sportlicher Belastung oder ein normaler Wundheilungsprozess, durch wIRA noch verbessert [12-14, 16-18, 23, 53]. Entsprechend breit sind die klinischen Anwendungsmöglichkeiten von wIRA.

\section{Allgemeine Anwendungsaspekte}

wIRA ist ein kontaktfreies, verbrauchsmaterialfreies, leicht anzuwendendes, (selbst bei Wunden) als angenehm empfundenes Verfahren mit guter Tiefenwirkung und anhaltendem Wärmedepot $[12,13,16-18]$. wIRA ist kreislaufschonend (verglichen mit warmem Vollbad), sauber (verglichen mit Fango), bei verschiedensten Lagerungen einsetzbar und benötigt keine Fixierung (verglichen mit warmem Wickel). wIRA bietet Bewegungsfreiheit, eine simultane Kombination mit Bewegung ist möglich. wIRA ist v. a. durch Variation des Bestrahlungsabstands und damit der Bestrahlungsstärke sowie über die Bestrahlungsdauer sehr gut dosierbar.

wIRA wird meist lokal/körperregionbezogen (lokoregional) und seltener systemisch auf den gesamten Körper wirkend (Ganzkörperhyperthermie) eingesetzt $[12,13,17,18]$. Entsprechend fokussiert die hier vorliegende Übersicht auf die lokoregionale Anwendung von wIRA.

Die Bestrahlung der unbedeckten Haut oder Wunde erfolgt senkrecht zur Haut mit einem wIRA-Strahler täglich 1-mal oder mehrmals über jeweils mind. (20-)30 min oder auch deutlich län- ger (mehrere Stunden pro Tag, z. B. Nachtstunden nutzend) $[19,23,53]$ mit moderater, als angenehm empfundener Bestrahlungsstärke (für allgemeine Anwendungen (ohne Wunden) typischerweise $60-120 \mathrm{~mW} / \mathrm{cm}^{2}$ wIRA bzw. $80-160 \mathrm{~mW} / \mathrm{cm}^{2}$ wIRA und sichtbares Licht VIS) $[12,13,15,17,18]$. Der Bestrahlungsabstand ist immer so zu wählen, dass die Bestrahlungsstärke als angenehm empfunden wird („Wohlfühlabstand“; bei derzeit üblichen wIRAStrahlern meist ca. 35-80 cm). Ein größerer Bestrahlungsabstand und eine geringere Bestrahlungsstärke sind zu wählen bei Patienten mit gestörtem Sensorium (z. B. diabetischer Polyneuropathie) oder gestörter Rückäußerungsfähigkeit, bei schlecht durchblutetem Gewebe, bei kaltem Gewebe oder geringem Unterhautgewebe (z. B. Schienbeinkante) $[23,53]$. Bei adäquater (moderater) Bestrahlungsstärke kann die Anwendung von wIRA als sicher angesehen werden und unerwünschte Effekte (wie thermisch bedingte Hautreizungen) können vermieden werden [23] (in [23] auch ausführlichere Erläuterungen hierzu).

Es sollte lieber länger mit moderater Bestrahlungsstärke als kürzer mit höherer Bestrahlungsstärke mit wIRA bestrahlt werden $[23,53]$.

In der Regel können wIRA-Effekte durch längere Einzelbestrahlungsdauern und größere tägliche Bestrahlungsdauern - bei immer moderater Bestrahlungsintensität - gesteigert werden.

wIRA geht nicht in relevantem Maß durch blickdichte Kleidung oder Verbände [12, 13, 17, 18].

\section{Wunden}

Aufgrund von 7 prospektiven klinischen Studien (davon 6 randomisierte kontrollierte Studien) sind die im Folgenden zusammengefassten Wirkungen von wIRA auf die Wundheilung gut belegt (überwiegend mit Evidenzgrad 1a, im Sinne von mehreren prospektiven randomisierten kontrollierten Studien, bzw. Ib), und zwar auf verschiedenartige Wunden (Operationswunden, Verbrennungswunden, chronische Unterschenkelulzera vorwiegend venöser Genese mit und ohne Diabetes mellitus) und anhand verschiedener, sich ergänzender Zielvariablen und Verfahren [21-23, 35, 53]:

- akute Schmerzminderung während einer wIRA-Bestrahlung (Evidenzgrad 1b): ausnahmslose akute Schmerzminderung bei 230 einzelnen Bestrahlungen, 18,5 vs. 0,0 auf einer VAS-Skala 0-100, p<0,000001, > Abb. 2 (Studie Heidelberg) [7, 17],

- Minderung der erforderlichen Schmerzmedikation (Evidenzgrad 1a/1b): Operationswunden: 52-69\% weniger Analgetikabedarf, $\mathrm{p}=$ 0,000020 bzw. 0,00037 bzw. 0,0045, > Abb. 3 (Studie Heidelberg) [7, 17]. Unterschenkelulzera: 6 vs. 14,5 Tbl., p = 0,000002 (Studie Basel) [17], zudem Studie Troms $ø /$ Hillerød [32],

- größere/schnellere Wundflächenreduktion, schnellere Epithelialisierung (Evidenzgrad 1a/1b): Verbrennungen: $90 \%$ Wundflächenreduktion nach 9 vs. 13 Tagen, $p=0,000011$,

- Abb. 4 (Studie Kassel) [8, 17]; Unterschenkelulzera: kompletter Verschluss nach 14 vs. 42 Tagen, $p=0,000005$ (Studie Basel) $[17,50]$,

- bessere Gesamteinschätzung der Wundheilung (Evidenzgrad 1a/1b): Operationswunden: 88,6 vs. 78,5 auf einer VAS-Skala $0-100, p<0,000001$ (Studie Heidelberg) $[7,17]$. Unterschen- 


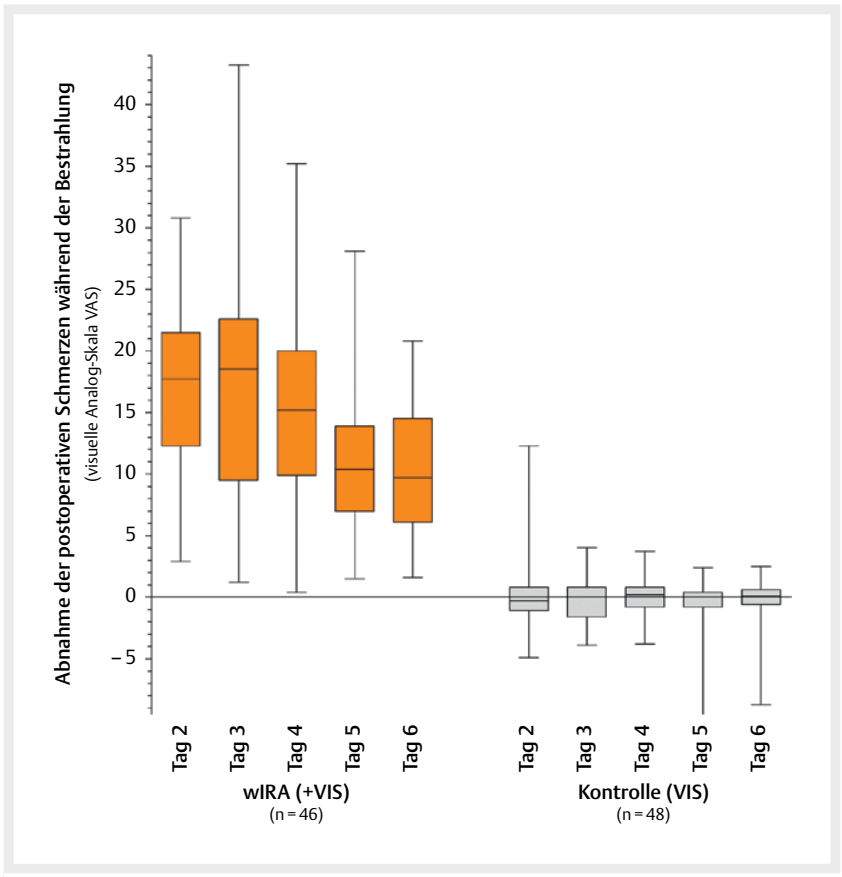

- Abb. 2 Abnahme der postoperativen Schmerzen während Bestrahlung in der Gruppe mit wassergefiltertem Infrarot A (wIRA) und sichtbarem Licht (VIS) und in der Kontrollgruppe mit nur sichtbarem Licht (VIS) (Abdominaloperationen, Studie Heidelberg) [16].

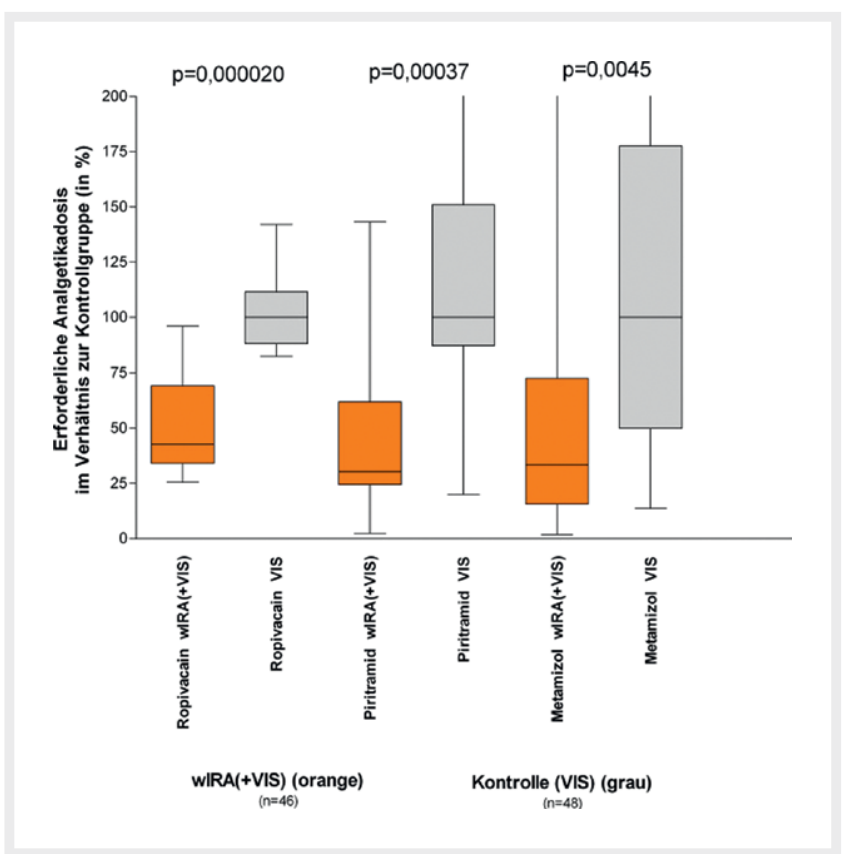

Abb. 3 Erforderliche Analgetikadosis in den Untergruppen mit wassergefiltertem Infrarot A (wIRA) und sichtbarem Licht (VIS) im Verhältnis zu den Kontrolluntergruppen mit nur sichtbarem Licht (VIS) (Mediane der Kontrolluntergruppen=100) (Studie Heidelberg) [16]. kelulzera: 85 vs. 67,5 auf einer VAS-Skala $0-100, p=0,012$ (Studie Freiburg) $[18,44]$,

- bessere Gesamteinschätzung des Effekts der Therapie (Evidenzgrad 1b): 79,0 vs. 46,8 auf einer VAS-Skala 0-100 mit 50 als Neutralpunkt, $p<0,000001$ (Studie Heidelberg) [7, 17],

- höherer Gewebesauerstoffpartialdruck während wIRA-Bestrahlung (Evidenzgrad 1b): in einer Gewebetiefe von $2 \mathrm{~cm}$ 41,6 vs. $30,2 \mathrm{mmHg}, \mathrm{p}<0,000001$, > Abb. 5 a (Studie Heidelberg) $[7,17]$,

- höhere Gewebetemperatur während wIRA-Bestrahlung (Evidenzgrad 1b): in einer Gewebetiefe von $2 \mathrm{~cm} 38,9$ vs. $36,4^{\circ} \mathrm{C}, \mathrm{p}<0,000001, \vee$ Abb. $\mathbf{5 b}$ (Studie Heidelberg) [7, 17],

- besserer kosmetischer Aspekt (Evidenzgrad 1b): 84,5 vs. 76,5 auf einer VAS-Skala 0-100, $p=0,00027$ (Studie Heidelberg) $[7,17]$,

- niedrigere Wundinfektionsrate (Evidenzgrad 1b): einmalige präoperative Bestrahlung: 5,1\% (9 von 178) vs. $12,1 \%$ (22 von 182) Wundinfektionen insgesamt, $p=0,017$; späte Wundinfektionen (an den postoperativen Tagen 9 bis 30): 1,7\% (3 von 178 ) vs. $7,7 \%$ (14 von 182), $p=0,007$ (Studie München) ([28] sowie aus den Daten der Publikation berechnete Werte [23]). Postoperative Bestrahlung: $7 \%$ (3 von 46) vs. $15 \%$ (7 von 48), $p=0,21$, Trend; späte Wundinfektionen $0 \%(0$ von 46$)$ vs. $8 \%$ (4 von 48), $p=0,12$, Trend (Studie Heidelberg) $[7,17]$,

- schnellere Granulation (Evidenzgrad 1b): 90 vs. 80 auf einer VAS-Skala 0-100, p=0,036 (Studie Freiburg) $[18,44]$,

- geringere Exsudation (Evidenzgrad 1b, Trend): 30 vs. 55 auf einer VAS-Skala 0-100, $p=0,075$, Trend (Studie Freiburg) [18,44],

- geringere Wundbeläge (Evidenzgrad 1b, Trend): 20 vs. 40 auf einer VAS-Skala 0-100, p=0,070, Trend (Studie Freiburg) $[18,44]$,

- kürzerer Krankenhausaufenthalt (Evidenzgrad 1b, Trend): 9 vs. 11 Tage, $p=0,022$, Trend (Studie Heidelberg) $[7,17]$.

Einen Überblick über weitere Details zu den klinischen Studien zu wIRA bei Wunden (auch mit der hier verwendeten Bezeichnung der Studien anhand der Studienorte), wie Bestrahlungszeiten und Bestrahlungshäufigkeiten, bietet die Publikation [23].

Die Effekte von wIRA bei Wunden wurden in den bisherigen Studien aufgrund begrenzter täglicher Bestrahlungszeiten (9-40 min) eher unterschätzt [23].

Die Anwendung bei Wunden beschränkt sich nicht auf Wundheilungsstörungen, da auch die „normale“ ungestört ablaufende Wundheilung von wIRA profitiert (schneller, schmerzärmer, mit sehr gutem kosmetischem Ergebnis),

Zumindest während der Bestrahlungen setzt die Anwendung von wIRA eine offene Wundbehandlung voraus [53].

In der Regel ist die unbedeckte Wunde täglich mind. insgesamt 60 min (oder auch deutlich länger, z. B. 2-6 Stunden pro Tag) mit moderater Bestrahlungsstärke (individuell ermittelter „Wohlfühlabstand“) zu bestrahlen (bei Wunden gilt orientierend: $70 \mathrm{~mW} / \mathrm{cm}^{2}$ für die Gesamtbestrahlungsstärke wIRA + VIS; bei Wunden mit verminderter Wärmeverträglichkeit des bestrahlten Gewebes gilt orientierend: $35 \mathrm{~mW} / \mathrm{cm}^{2}$ für die Gesamtbestrahlungsstärke wIRA + VIS). Es sollte bis zum Abschluss der Wundheilung und lieber öfter und länger mit geringerer Bestrahlungsstärke als kürzer mit höherer Bestrahlungsstärke bestrahlt werden [23, 53]. 


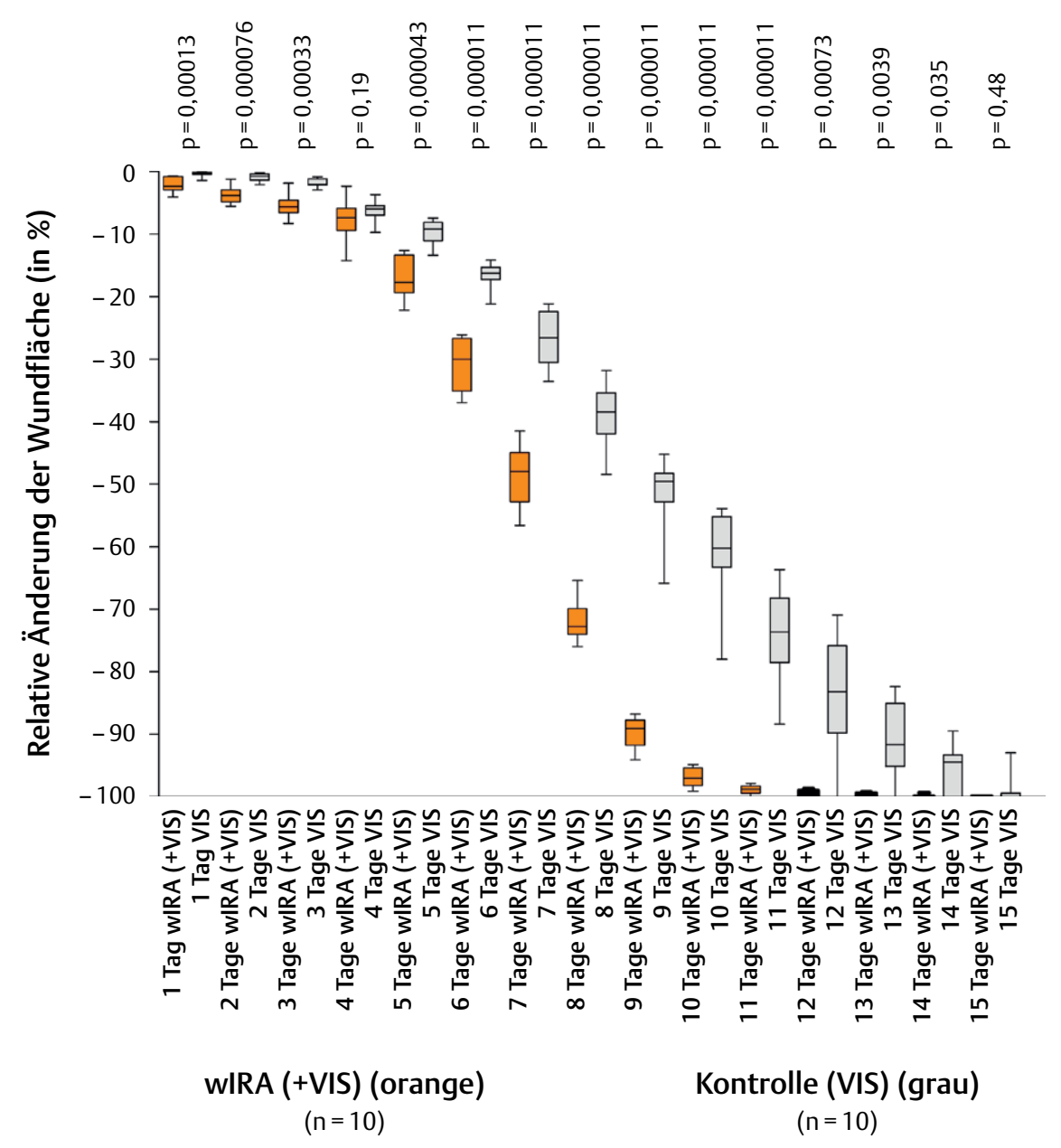

- Abb. 4 Relative Änderung der Wundfläche von schwerbrandverletzten Kindern in Abhängigkeit von der Dauer der Behandlung (in Tagen) in der Gruppe mit wassergefiltertem Infrarot A (wIRA) und sichtbarem Licht (VIS) und in der Kontrollgruppe mit nur sichtbarem Licht (VIS) (Studie Kassel) [16].

Die $>$ Abb. 6 zeigt den Verlauf einer Wundinfektion unter Therapie mit wIRA [53].

Erfahrungen mit wIRA bei Wunden außerhalb von Studien:

wIRA ist auch bei Dekubitalulzera sowohl präventiv als auch therapeutisch (Verkleinerung der Wundfläche, bessere Granulation) einsetzbar [16-18, 50].

wIRA kann beim diabetischen Fuß sowohl präventiv - bei gefährdeten Patienten mit Diabetes mellitus und Sensibilitätsstörungen - vor dem Auftreten einer Wunde als auch therapeutisch eingesetzt werden $[17,18]$.

Zusammenfassend: wIRA stellt eine wertvolle Therapieoption dar und kann für die Behandlung von akuten und chronischen Wunden, auch infizierten Wunden, und Verbrennungen - eingebettet in ein Gesamttherapiekonzept - empfohlen werden [23, 53].

\section{Hauterkrankungen}

Vulgäre Warzen können mit 6-9 einwöchigen Therapiezyklen mit kontinuierlicher Keratolyse mit Salizylsäurepflaster und jeweils einer unblutigen Kürettage und einer Bestrahlung mit wIRA und sichtbarem Licht VIS (diese Kombination ist bei wIRA-Strahlern üblich) von 30 min erfolgreich therapiert werden $[5,14,17,18]$. In einer prospektiven, randomisierten, kontrollierten, doppeltblinden Studie der Hautklinik der Universität Jena mit 80 Patienten mit therapierefraktären Warzen wurden bei nur drei 3-wöchigen Therapiezyklen (somit nur insgesamt 3 Bestrahlungen) in den beiden Gruppen mit wIRA(+ VIS) eine Minderung der Gesamtwarzenfläche pro Patient im Median um 94\%/99\% im Vergleich zu 47\%/73\% in den beiden Gruppen mit nur VIS sowie $72 \%$ im Vergleich zu 34\% völlig verschwundene Warzen und $42 \%$ im Vergleich zu 7\% warzenfreie Patienten erreicht [5].

wIRA beschleunigt die Abheilung von Herpes labialis und mindert die Schmerzen bei Herpes Zoster sowohl akut als auch die chronischen Post-Zoster-Schmerzen [12-14, 17, 18].

wIRA bewirkt bei Sklerodermie eine Befindlichkeitsbesserung, weniger Schmerzen und eine Minderung der Raynaud-Symptomatik [1214, 17, 18]. Bei Morphea (und den anderen Formen der Sklerodermie) führt wIRA zu einer Rückbildung der Sklerose (mit messbarer Rückbildung von Hauthärte und Größe von Plaques) und Abnahme des Juckreizes und der Schmerzen [12-14, 17, 18, 49, 51]. Bei ulzerierter Morphea kann eine Abheilung des Ulkus erreicht werden [6, 18].

Bei Akne papulopustulosa führt eine Therapie mit wIRA und dem vollen sichtbaren Spektrum v. a. zu einer Reduktion der Ent- 


\section{a \\ a Sauerstoffpartialdruck $\mathrm{pO}_{2}$}

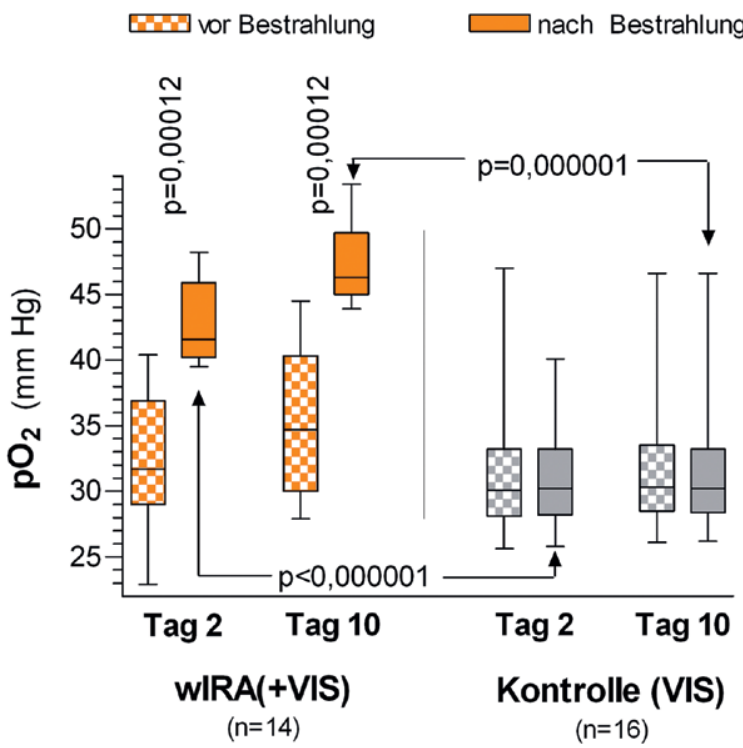

b
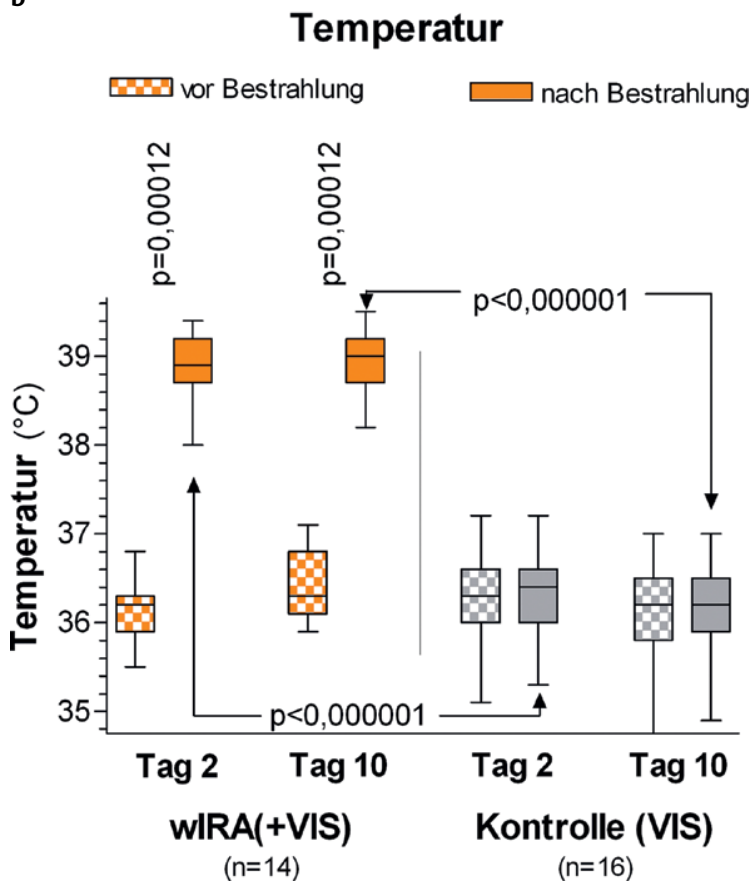

Abb. 5 Sauerstoffpartialdruck und Temperatur in $2 \mathrm{~cm}$ Gewebetiefe an den postoperativen Tagen 2 und 10 in der Gruppe mit wassergefiltertem Infrarot A (wIRA) und sichtbarem Licht (VIS) und in der Kontrollgruppe mit nur sichtbarem Licht (VIS) (Abdominaloperationen, Studie Heidelberg) [16].

zündungszeichen sowie der Seborrhoe [14, 17-19]. Eine Kombination mit einem Dermatikum ist möglich.

wIRA ist zur Resorptionsverbesserung topischer Dermatika und Substanzen (z. B. Cortison, Lokalanästhetika) als Alternative zum Okklusivverband einsetzbar [9, 14, 17, 18, 39], um z. B. bei Psoria- sis oder bei Neurodermitis weniger Cortison oder ein weniger starkes Cortison zu verwenden.

wIRA vermag im Rahmen einer Photodynamischen Therapie (PDT) zusammen mit einer oder mehreren Anregungsbanden (Wirkbanden) im sichtbaren Bereich (VIS) und einem topisch aufgetragenen Photosensibilisator (oder einer Vorstufe hiervon) bei aktinischen Keratosen eingesetzt zu werden [4, 14, 17, 18, 26, 48]. Gegenüber anderen für PDT verwendeten Bestrahlungsquellen ist wIRA(+ VIS) schmerzärmer $[14,17,18,48]$.

\section{Physiotherapie, Orthopädie und Sportmedizin}

Im Hinblick auf Schmerz- und Entzündungsminderung sowie Durchblutungssteigerung ist wIRA präventiv, therapeutisch, regenerativ oder rehabilitativ einsetzbar bei muskulären Verspannungen (z. B. Schulter-Nacken-Verspannungen), Myogelosen [24] (muskelentspannender Effekt der Wärme), Lumbago, Erkrankungen des rheumatischen Formenkreises [29], ankylosierender Spondylitis (Morbus Bechterew) [3, 30], Arthrosen (Knie-, Hüft-, Fingergelenkarthrosen, hierzu auch kontrollierte Studie [33]), Arthritiden (entzündungsmindernder Effekt; hierzu auch kontrollierte Studie bei Psoriasisarthritis [31]), Kontusionen und in der postoperativen Rehabilitation [12-14, 16, 17]. Die Resorption von Hämatomen und Seromen wird durch wIRA beschleunigt $[8,16,17]$.

Zum sportmedizinischen Bereich liegen u. a. 6 Studien mit wIRA vor [20].

wIRA lässt sich zur muskulären Regeneration nach Sport [11,17] (wIRA in Ruhe oder wIRA in Kombination mit Bewegung, s. u.) verwenden: Nach stufenweise ansteigender ausbelastender Ergometrie verbesserte sich bei 25 Probanden einer prospektiven, randomisierten, kontrollierten Studie während anschließender Bestrahlung der ventralen Oberschenkelmuskulatur mit wIRA(+ VIS) in Ruhe über 20 min (Tag mit Bestrahlung) das Befinden der Muskulatur auf einer visuellen Analogskala (0-100) von 36 auf 71 signifikant mehr $(p=0,0138$ ) als in Ruhe ohne Bestrahlung (von 34 auf 54, Kontrolltag) und erreichte nach Bestrahlung bemerkenswerterweise binnen 20 min wieder den Ausgangswert vor Ergometrie von 70. Die Leistungsfähigkeit sank von der ersten Ergometrie zu der sich nach den 20 min anschließenden zweiten Ergometrie am Tag mit Bestrahlung signifikant weniger $(p=0,0128)$ als am Kontrolltag $[11,14,17,20]$.

\section{Simultane Kombination mit Bewegung}

Da wIRA - im Gegensatz zu anderen wärmeapplizierenden Verfahren wie Fango oder heiße Rolle - ein kontaktfreies Verfahren ist, lässt sich wIRA simultan mit Bewegung kombinieren [14, 17, 34].

In einer prospektiven, randomisierten, kontrollierten Studie mit 40 adipösen Frauen verminderte sich die „Summe der Umfänge von Taille, Hüfte und beiden Oberschenkeln von jeder Probandin “ während 4 Wochen signifikant mehr $(p<0,001)$ in der Gruppe mit wIRA (während Ergometertraining) als in der unbestrahlten Kontrollgruppe (nur Ergometertraining) [14, 17, 20, 34]. Auch nahm das Körpergewicht während der 4 Wochen in der Gruppe mit wIRA deutlich mehr als in der Kontrollgruppe ab. Die Ergebnisse der Studie legen nahe, dass wIRA 

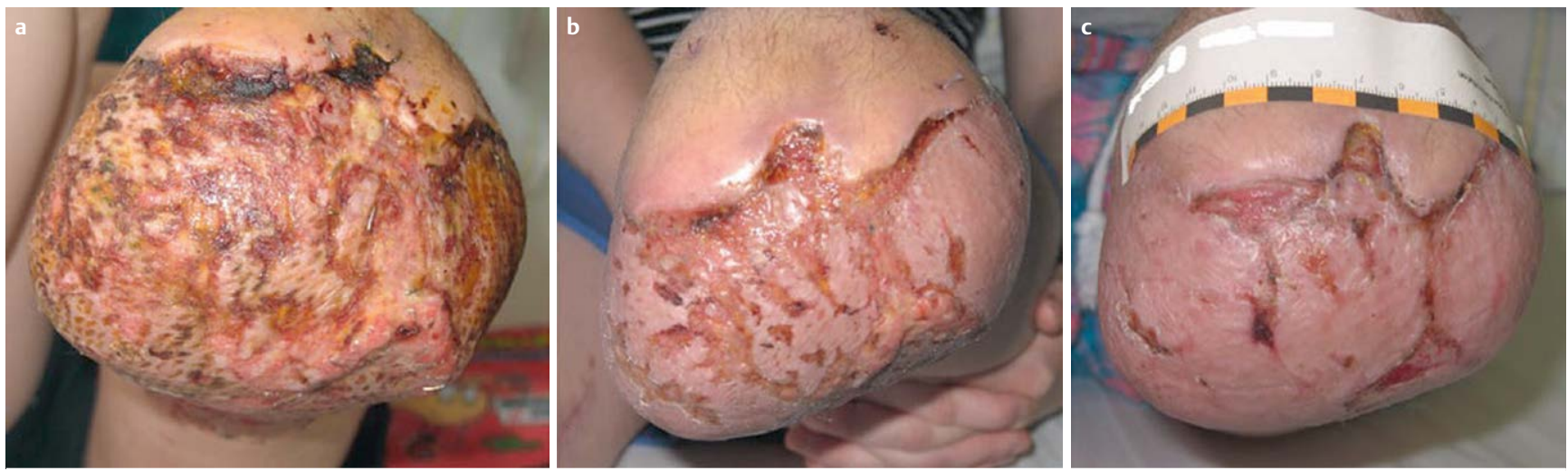

- Abb. 6 a Fortschreitende Auflösung transplantierter Spalthautgitter wegen Infektion mit „Extended-spectrum“- $\beta$-Laktamase (ESBL) bildenden Klebsiellen, Ansicht der Stumpfkuppe (Femur), 9 Tage postoperativ. b 4 Tage nach Verlegung und Beginn der Bestrahlungen mit wIRA, 3-mal täglich 1 h. c Fortschreitende Epithelialisierung 3 Wochen nach Beginn der Bestrahlungen mit wIRA [53]

- während moderater Fußkurbelergometer-Ausdauerbelastung als lipolytischem Reiz - die lokale Lipolyse (speziell am Oberschenkel) in dem sonst bradytrophen und hypothermen Fettgewebe steigert und die mobilisierten Fette in der Muskulatur während der Ergometerbelastung verbrannt werden. Dies ist nutzbar, um in Verbindung mit einer angemessenen Ernährung die Körperzusammensetzung, insbesondere die lokale Fettverteilung, und die Abnahme von Fett und Körpergewicht bei adipösen Personen zu verbessern.

Auch zur Therapie der Fibromyalgie lässt sich die Kombination von wIRA(+VIS) mit Bewegung (Ergometer und mehrere wIRAStrahler) mit hierbei nur niedriger Belastung erfolgreich zur Schmerzminderung nutzen $[12-14,17,20]$.

\section{Neonatologie}

wIRA wird bei Neugeborenen zur Aufrechterhaltung oder Erhöhung der Körpertemperatur eingesetzt [17,47]. wIRA vermag vor einem Transport ein „Wärmedepot“ aufzubauen, was einer Auskühlung auf dem anschließenden Transport entgegenwirkt, sodass die Häufigkeit erniedrigter Körpertemperatur (Hypothermie-Inzidenz) der Neugeborenen bei Aufnahme auf die Intensivstation verringert wird. wIRA reduziert den Körpertemperaturabfall bei Frühgeborenen während Inkubatorpflege.

\section{Anästhesiologie und Intensivmedizin}

In der Intensivmedizin vermag wIRA z. B. eine Hypersekretion bei Bronchopneumonien zu mindern und die pulsoximetrisch messbare Hämoglobin-Sauerstoff-Sättigung durch thorakale Bestrahlung mit wIRA bei Verschleimung infolge gestörten Schluckens und Abhustens anzuheben [17].

wIRA lässt sich zur postoperativen Wiedererwärmung z. B. nach kardiochirurgischen Eingriffen verwenden [2, 10, 17].

Im Bereich der Viszeralchirurgie und der Intensivmedizin vermögen wIRA-Bestrahlungen des Abdomens die Darmmotilität zu aktivieren [17].

\section{Neurologie}

wIRA-Bestrahlungen der Kehlkopf- und Halsregion lassen sich zur Verbesserung der Sprech- und Schluckfunktion bei z. B. postoperativen Sprech- und Schluckstörungen einsetzen, soweit eine periphere Beeinflussbarkeit gegeben erscheint [17].

wIRA ist mit niedrigen Bestrahlungsstärken und beginnend mit kurzen Bestrahlungszeiten zur Therapie des komplexen regionalen Schmerzsyndroms (Complex Regional Pain Syndrom CRPS) einsetzbar [17].

wIRA vermag auch die Symptome von Polyneuropathien verschiedener Genese (zytostatikabedingt, idiopathisch) deutlich zu mindern [17].

\section{Hals-Nasen-Ohren (HNO)}

wIRA lässt sich gut zur Therapie von Sinusitiden und Laryngitiden einsetzen.

\section{Onkologie}

wIRA zur lokalen Hyperthermie oder systemischen Hyperthermie (Ganzkörperhyperthermie) kann mit Strahlentherapie (z. B. beim metastasierenden Mamma-Karzinom) oder Chemotherapie kombiniert werden [12-14, 17].

Hyperthermie wird in mehrerlei Hinsicht als komplementär zur Strahlentherapie angesehen $[17,43]$ : ionisierende Strahlung wirkt vorwiegend auf die M- und G1-Phase des Zellzyklus, während Hyperthermie v. a. auf die S-Phase wirkt; Strahlentherapie ist in alkalischem Gewebe sehr effektiv, während die Zytotoxizität der Hyperthermie unter sauren Bedingungen verstärkt ist; Strahlentherapie wirkt nicht effektiv auf hypoxisches Gewebe, während Hyperthermie sehr zytotoxisch auf hypoxische Zellen wirkt. Durch die Kombination von Hyperthermie und Strahlentherapie lässt sich sowohl der hypoxische, einen niedrigen (sauren) pH aufweisende Tumorkern wie auch der relativ gut perfundierte äußere Teil des Tumors behandeln. Zudem erhöht die Hyperthermie über ihre vaskulären Effekte (verstärkte Perfusion) die Tumoroxygenation, was die Wirkung der Strahlentherapie verstärkt. Hyperthermie erhöht außerdem die Produktion von Sauerstoffradikalen während Strah- 
lentherapie und vermindert die Reparatur von strahlentherapiebedingten DNA-Schäden. So haben Hyperthermie und Strahlentherapie oft einen synergistischen Effekt.

Bereits in den 1990er Jahren wurde lokale wIRA-Bestrahlung, über Temperaturmessfühler geregelt, zur lokalen Hyperthermie in Kombination mit Strahlentherapie erfolgreich z. B. bei weit über 100 Patientinnen mit lokal metastasierendem Mammakarzinom eingesetzt [13, 17, 45, 46, 52]. 2009 wurde dieses Konzept mit wesentlich verfeinerter Mess- und Regeltechnik wieder aufgegriffen: Es wird jeweils zunächst die lokale thermografie-geregelte wIRAHyperthermie und dann die Strahlentherapie durchgeführt, sodass durch die wIRA-Hyperthermie die Tumoroxygenation erhöht ist, was die Wirkung der Strahlentherapie verstärkt [17, 37, 38]. Über die kontinuierliche Thermografie und deren automatische quantitative Auswertung wird die wIRA-Bestrahlung dabei so geregelt, dass ein Maximum an Hyperthermie ohne Überschreitung individuell einstellbarer Grenzwerte erreicht wird.

\section{FAZIT}

Die Anwendungsbreite von wIRA ist im Hinblick auf die indikationsübergreifende Minderung von Schmerzen, Entzündung und vermehrter Sekretion und die Förderung von Infektionsabwehr und Regeneration groß. Dabei sollte wIRA immer eingebettet in ein Gesamttherapiekonzept gesehen werden. Dann ist wIRA, das den großen Vorteil besitzt, ein substanzfreies Verfahren darzustellen, das bei angemessener Anwendung keine unerwünschten Wirkungen aufweist, vielfältig sinnvoll einsetzbar.

Weitere Fachinformationen zu wassergefiltertem Infrarot A (wIRA): http://www.waerme-therapie.com/fachartikel.html

\section{Interessenkonflikterklärung}

Der Autor ist für die Dr. med. h.c. Erwin Braun Stiftung, Basel, Schweiz, eine gemeinnützige eidgenössisch anerkannte Schweizer Wissenschaftsstiftung, tätig. Die Stiftung war nicht in die Erstellung der Übersicht involviert. Der Autor ist nicht und war nicht bei einer Firma angestellt und erhielt/erhält auch keine Zuwendungen einer Firma. Deshalb erklärt der Autor, dass kein Interessenskonflikt im Sinne der Richtlinien des International Committee of Medical Journal Editors besteht.

Literatur

[1] Akca O, Melischek M, Scheck T et al. Postoperative pain and subcutaneous oxygen tension. Lancet 1999; 354: 41-42 PMID 10406365 doi:10.1016/S0140-6736(99)00874-0

[2] Bräuer A, Weyland W, Kazmaier S et al. Efficacy of postoperative rewarming after cardiac surgery. Ann Thorac Cardiovasc Surg 2004; 10: 171-177

[3] Falkenbach A, Dorigoni H, Werny F et al. Wassergefilterte Infrarot-ABestrahlung bei Morbus Bechterew und degenerativen Wirbelsäulenveränderungen: Effekte auf Beweglichkeit und Druckschmerzhaftigkeit. Österr Z Physikal Med Rehab 1996; 6: 96-102
[4] Foss P. Einsatz eines patentierten, wassergefilterten Infrarot-A-Strahlers (Hydrosun) zur photodynamischen Therapie aktinischer Dyskeratosen der Gesichts- und Kopfhaut. Z naturheilkundl Onkologie krit Komplementärmed 2003; 6: 26-28

[5] Fuchs SM, Fluhr JW, Bankova L et al. Photodynamic therapy (PDT) and waterfiltered infrared $A(w I R A)$ in patients with recalcitrant common hand and foot warts. Ger Med Sci 2004; 2: Doc08 http://www.egms. de/static/pdf/journals/gms/2004-2/000018.pdf http://www.egms.de/ static/en/journals/gms/2004-2/000018.shtml

[6] Geißler E, Schumann H. (2009) Wassergefiltertes Infrarot A (wIRA) bei ulzerierter Morphea. Z Wundheilung 2004; 13: 177-180

[7] Hartel M, Hoffmann G, Wente MN et al. Randomized clinical trial of the influence of local water-filtered infrared $A$ irradiation on wound healing after abdominal surgery. Br J Surg 2006; 93: 952-960 doi:10.1002/bjs.5429

[8] Hartel M, Illing P, Mercer JB et al. Therapy of acute wounds with water-filtered infrared-A (wIRA) (review). GMS Krankenhaushyg Interdiszip 2007; 2: Doc53 http://www.egms.de/pdf/journals/ dgkh/2007-2/dgkh000086.pdf http://www.egms.de/static/en/ journals/dgkh/2007-2/dgkh000086.shtml

[9] Haupenthal H. In vitro- und in vivo-Untersuchungen zur temperaturgesteuerten Arzneistoff-Liberation und Permeation. Mainz: Johannes Gutenberg-Universität; Diss. 1997

[10] Hellige G, Trostdorf U, Weyland W. Effektivität eines Infrarot-A-Strahlers mit Wasserfilter zur postoperativen Wiedererwärmung kardiochirurgischer Patienten. In: Vaupel P, Krüger W, (Hrsg.). Wärmetherapie mit wassergefilterter Infrarot-A-Strahlung. Grundlagen und Anwendungsmöglichkeiten. 2. Aufl. Stuttgart: Hippokrates; 1995: 157-165

[11] Hoffmann G. Improvement of regeneration by local hyperthermia induced by waterfiltered infrared A (wIRA). Int J Sports Med 2002; 23: (Suppl 2): S145

[12] Hoffmann G. Wassergefiltertes Infrarot A (wIRA) zur Verbesserung der Wundheilung (Übersichtsarbeit). GMS Krankenhaushyg Interdiszip 2006; 1: Doc20 http://www.egms.de/static/pdf/journals/dgkh/20061/dgkh000020.pdf http://www.egms.de/static/en/journals/ dgkh/2006-1/dgkh000020.shtml

[13] Hoffmann G. Principles and working mechanisms of water-filtered infrared-A (wIRA) in relation to wound healing (review). GMS Krankenhaushyg Interdiszip 2007; 2: Doc54 http://www.egms.de/pdf/ journals/dgkh/2007-2/dgkh000087.pdf http://www.egms.de/static/ en/journals/dgkh/2007-2/dgkh000087.shtml

[14] Hoffmann G. Klinische Anwendungen von wassergefiltertem Infrarot A (wIRA). In: Kaase H, Serick F (Hrsg.). Sechstes Symposium „Licht und Gesundheit“. Eine Sondertagung der Technischen Universität Berlin und der Deutschen Gesellschaft für Photobiologie mit der Deutschen Akademie für Photobiologie und Phototechnologie und der Deutschen Lichttechnischen Gesellschaft, Berlin, 13./14.03.2008. Berlin: 2008: 130-146 ISBN 3-9807635-0-3. Auch online frei verfügbar http:// publikationen.ub.uni-frankfurt.de/frontdoor/index/index/docld/59

[15] Hoffmann G. Wassergefiltertes Infrarot A (wIRA) zur Verbesserung der Wundheilung bei akuten und chronischen Wunden. Wundmanagement 2008; 2: 72-80 Auch online frei verfügbar http://publikationen. ub.uni-frankfurt.de/volltexte/2008/5429/

[16] Hoffmann G. Water-filtered infrared-A (wIRA) in acute and chronic wounds (bilingual review). Wassergefiltertes Infrarot A (wIRA) bei akuten und chronischen Wunden (zweisprachige Übersichtsarbeit). GMS Krankenhaushyg Interdiszip 2009; 4: Doc12 doi:10.3205/dgkh000137 http://www.egms.de/pdf/journals/dgkh/2009-4/dgkh000137.pdf http:// www.egms.de/static/en/journals/dgkh/2009-4/dgkh000137.shtml

[17] Hoffmann G. Wassergefiltertes Infrarot A in Chirurgie, Dermatologie, Sportmedizin und weiteren Bereichen. In: Krause R, Stange R (Hrsg.). Lichttherapie. Berlin, Heidelberg, New York, Tokio: Springer; 2012: 25-54 Auch online frei verfügbar http://publikationen.ub.unifrankfurt.de/frontdoor/index/index/docld/24171 
[18] Hoffmann G. Wassergefiltertes Infrarot A (wIRA) in Chirurgie und Dermatologie. In: Völker S (Hrsg.). Siebentes Symposium „Licht und Gesundheit". Eine Sondertagung der Technischen Universität Berlin und der Deutschen Akademie für Photobiologie und Phototechnologie (DAfP), der Deutschen Gesellschaft für Photobiologie (DGP) und der Deutschen Lichttechnischen Gesellschaft (LiTG), 15.-16.03.2012 in Berlin. Berlin: Universitätsverlag der Technischen Universität Berlin, Universitätsbibliothek; 2012: 202-231 Auch online frei verfügbar http://publikationen.ub.uni-frankfurt.de/frontdoor/index/index/ docld/22797

[19] Hoffmann G. Wassergefiltertes Infrarot A (wIRA) in der Dermatologie. Haut Dermatol Allergol Kosmetol 2013; 24: 228-229. Auch online frei verfügbar http://publikationen.ub.uni-frankfurt.de/frontdoor/index/ index/docld/32142

[20] Hoffmann G. Wassergefiltertes Infrarot A (wIRA) in der Sport- und Präventivmedizin. In: Völker S, Schumacher H (Hrsg.). 8. Symposium „Licht und Gesundheit“. Eine Sondertagung der TU Berlin gemeinsam mit der Deutschen Akademie für Photobiologie und Phototechnologie (DAfP) und der Deutschen Lichttechnischen Gesellschaft (LiTG), 19. und 20. März 2014, Berlin. Berlin: Universitätsverlag der TU Berlin; 2014: 80-105 Auch online verfügbar http://publikationen.ub. uni-frankfurt.de/frontdoor/index/index/docld/35166

[21] Hoffmann G. Wassergefiltertes Infrarot A (wIRA) fördert die Heilung von Verbrennungen und Wunden. Deutschsprachige Arbeitsgemeinschaft für Verbrennungsbehandlung. 34. Jahrestagung der Deutschsprachigen Arbeitsgemeinschaft für Verbrennungsbehandlung (DAV 2016). Berchtesgaden, Deutschland, 13.-16.01.2016. Düsseldorf: German Medical Science GMS Publishing House; 2016: Doc16dav47 doi:10.3205/16dav47 urn:nbn:de:0183-16dav479 http://www.egms. de/static/en/meetings/dav2016/16dav47.shtml

[22] Hoffmann G. Water-filtered infrared-A (wIRA) promotes wound healing. Deutsche Gesellschaft für Chirurgie. 133. Kongress der Deutschen Gesellschaft für Chirurgie. Berlin, 26.-29.04.2016. Düsseldorf: German Medical Science GMS Publishing House; 2016: Doc16dgch599 doi: 10.3205/16dgch599 urn:nbn:de:018316dgch5990 http://www.egms.de/static/de/meetings/ dgch2016/16dgch599.shtml

[23] Hoffmann G, Hartel M, Mercer JB. Heat for wounds - water-filtered infrared-A (wIRA) for wound healing - a review. GMS Ger Med Sci 2016; 14: Doc08 doi:10.3205/000235 URN: urn:nbn:de:0183-0002352 http://www.egms.de/static/pdf/journals/gms/2016-14/000235.pdf http://www.egms.de/static/en/journals/gms/2016-14/000235.shtml

[24] Hoffmann G, Siegfried I. Volkskrankheit Rückenschmerz: neue Sichtweisen. Seminar des Arbeitskreises Sportmedizin der Akademie für ärztliche Fortbildung und Weiterbildung der Landesärztekammer Hessen. Bad Nauheim, 05.06.2004. Düsseldorf, Köln: German Medical Science; 2005: Doc04ruecken1 http://www.egms.de/static/pdf/ meetings/ruecken2004/04ruecken1.pdf http://www.egms.de/static/ en/meetings/ruecken2004/04ruecken1.shtml

[25] Hopf HW, Hunt TK, West JM et al. Wound tissue oxygen tension predicts the risk of wound infection in surgical patients. Arch Surg 1997; 132: 997-1004 PMID 9301613

[26] Hübner K. Die Photo-dynamische Therapie (PDT) der aktinischen Keratosen, Basalzellkarzinome und Plantarwarzen. derm - Praktische Dermatologie 2005; 11: 301-304

[27] Karu TI. Multiple roles of cytochrome c oxidase in mammalian cells under action of red and IR-A radiation (critical review). IUBMB Life 2010; 62: 607-610 PMID 20681024

[28] Künzli BM, Liebl F, Nuhn P et al. Impact of preoperative local water-filtered infrared $A$ irradiation on postoperative wound healing: a randomized patient- and observer-blinded controlled clinical trial. Ann Surg 2013; 258: 887-894. doi:10.1097/SLA.00000000000000235
[29] Lange U. Infrarotes Spektrum - Muskuloskeletale Indikationen. In: Krause R, Stange R (Hrsg.). Lichttherapie. Berlin, Heidelberg, New York: Springer; 2012: 20-25; 49-50 ISBN 978-3-642-16938-0

[30] Lange U, Müller-Ladner U, Dischereit G. Wirkung iterativer Ganzkörperhyperthermie mit wassergefilterter Infrarot-A-Strahlung bei ankylosierender Spondylitis - eine kontrollierte, randomisierte, prospektive Studie. Akt Rheumatol 2017; 42: 122-128 doi:10.1055/ s-0042-116945

[31] Lange U, Schwab F, Müller-Ladner U et al. Wirkung einer iterativen Ganzkörperhyperthermie mit wassergefilterter Infrarot-A-Strahlung bei Arthritis psoriatica - eine kontrollierte, randomisierte prospektive Studie. Akt Rheumatol 2014; 39: 310-316

[32] Mercer JB, Nielsen SP, Hoffmann G. Improvement of wound healing by water-filtered infrared-A ( $w I R A$ ) in patients with chronic venous stasis ulcers of the lower legs including evaluation using infrared thermography. GMS Ger Med Sci 2008; 6: Doc11 und http://www.egms.de/pdf/ gms/2008-6/000056.pdf http://www.egms.de/static/en/journals/ gms/2008-6/000056.shtml

[33] Merle S. Wassergefilterte Infrarot-A-Strahlung zur Therapie der schmerzhaften Arthrose. In: Vaupel P, Krüger W (Hrsg): Wärmetherapie mit wassergefilterter Infrarot-A-Strahlung. Grundlagen und Anwendungsmöglichkeiten. 2. Aufl. Stuttgart: Hippokrates; 1995: 149-156

[34] Möckel F, Hoffmann G, Obermüller R et al. Influence of water-filtered infrared-A (WIRA) on reduction of local fat and body weight by physical exercise. GMS Ger Med Sci 2006; 4: Doc05 http://www.egms.de/ static/pdf/journals/gms/2006-4/000034.pdf http://www.egms.de/ static/en/journals/gms/2006-4/000034.shtml

[35] Müller W. Heat for wounds - water-filtered infrared-A (wIRA) assists wound healing. Press release of the Arbeitsgemeinschaft der Wissenschaftlichen Medizinischen Fachgesellschaften AWMF. Informationsdienst Wissenschaft idw, 30.06.2016. https://idw-online. de/de/news655385

[36] Niinikoski J, Gottrup F, Hunt TK. The role of oxygen in wound repair. In: Janssen $\mathrm{H}$, Rooman R, Robertson JIS (Hrsg.). Wound healing. Petersfield: Wrightson Biomedical Publishing; 1991: 165-174

[37] Notter M, Germond JF, Berz J et al. Thermographically guided irradiation with water-filtered infrared-A (wIRA) and radiotherapy in breast cancer recurrencies - First experiences and temperature analysis. Thermology Int 2011; 21: 47-53

[38] Notter M, Piazena H, Vaupel P. Hypofractionated re-irradiation of large-sized recurrent breast cancer with thermography-controlled, contact-free water-filtered infra-red-A hyperthermia: a retrospective study of 73 patients. Int J Hyperthermia 2017; 33: 227-236 [Epub ahead of print: 2016 Sep 28] PMID: 27618745 doi:10.1080/026 56736.2016.1235731

[39] Otberg N, Grone D, Meyer L et al. Water-filtered infrared-A (wIRA) can act as a penetration enhancer for topically applied substances. GMS Ger Med Sci 2008; 6: Doc08 http://www.egms.de/static/pdf/journals/ gms/2008-6/000053.pdf http://www.egms.de/static/en/journals/ gms/2008-6/000053.shtml

[40] Pediani R. What has pain relief to do with acute surgical wound healing? World Wide Wounds 2001 http://www.worldwidewounds. com/2001/march/Pediani/Pain-relief-surgical-wounds.html

[41] Piazena H. Vergleich der Spektren eines Strahlers für wassergefiltertes Infrarot A (wIRA, 1) und der Sonne in den Subtropen bei wolkenlosem Himmel am Mittag im Juni in Meeresspiegelhöhe (2) sowie beispielhaft von zwei unterschiedlichen Halogenstrahlern ohne Wasserfilterung für therapeutische und Wellnessanwendungen (3 und 4). 2013. http:// commons.wikimedia.org/wiki/File:WIRA-Wiki-GH-017C-de-SpektrenwIRA-Sonne-Halogenstrahler.png 
[42] Piazena H, Meffert $\mathrm{H}$, Uebelhack R et al. Thermische, schädigende und protektive Wirkungen von Infrarot-Hautbestrahlungen. In: Völker $S$ (Hrsg.). Siebentes Symposium „Licht und Gesundheit“. Eine Sondertagung der Technischen Universität Berlin und der Deutschen Akademie für Photobiologie und Phototechnologie (DAfP), der Deutschen Gesellschaft für Photobiologie (DGP) und der Deutschen Lichttechnischen Gesellschaft (LiTG), 15.-16.03.2012 in Berlin. Berlin: Universitätsverlag der Technischen Universität Berlin, Universitätsbibliothek; 2012: 161-182

[43] Rowe-Horwege RW. Hyperthermia, systemic. In: Webster JG (ed.). Encyclopedia of medical devices and instrumentation. Second edition. John Wiley \& Sons; 2006: 42-62

[44] Schumann H, Calow T, Weckesser $S$ et al. Water-filtered infrared A for the treatment of chronic venous stasis ulcers of the lower legs at home: a randomized controlled blinded study. $\mathrm{Br}$ J Dermatol 2011; 165: 541-551

[45] Seegenschmiedt M. Erfahrungen mit einem Infrarot-A-HyperthermieProjektor mit Wasserfilter zur lokal-perkutanen Hyperthermie kombiniert mit Radiotherapie bei oberflächennahen Tumoren. In: Vaupel P, Krüger W (Hrsg.). Wärmetherapie mit wassergefilterter Infrarot-A-Strahlung. Grundlagen und Anwendungsmöglichkeiten. 1. Aufl. Stuttgart: Hippokrates; 1992: 63-76

[46] Seegenschmiedt M, Leemann B, Walther E et al. Multizentrische Phase I/II-Studie zur Kombination von wassergefilterter Infrarot-A-Hyperthermie und Radiotherapie bei fortgeschrittenen und rezidivierten Tumoren. In: Vaupel P, Krüger W (Hrsg.). Wärmetherapie mit wassergefilterter Infrarot-A-Strahlung. Grundlagen und Anwendungsmöglichkeiten. 2. Aufl. Stuttgart: Hippokrates; 1995: 167-181

[47] Singer D, Schröder M, Harms K. Vorteile der wassergefilterten gegenüber herkömmlicher Infrarot-Strahlung in der Neonatologie. Z Geburtshilfe Neonatol 2000; 204: 85-92 PMID 10909163
[48] von Felbert V, Hoffmann G, Hoff-Lesch S et al. Photodynamic therapy of multiple actinic keratoses: Reduced pain through use of visible light plus water-filtered infrared-A (wIRA) compared to light from light-emitting diodes. Br J Dermatol 2010; 163: 607-615 doi:10.1111/ j.1365-2133.2010.09817.x

[49] von Felbert V, Kernland-Lang K, Hoffmann G et al. Irradiation with water-filtered infrared-A (wIRA) plus visible light improves cutaneous scleroderma lesions in a series of cases. Dermatology 2011; 222: 347-357

[50] von Felbert V, Schumann H, Mercer JB et al. Therapy of chronic wounds with water-filtered infrared-A (wIRA) (review). GMS Krankenhaushyg Interdiszip 2007; 2: Doc52 http://www.egms.de/pdf/journals/ dgkh/2008-2/dgkh000085.pdf http://www.egms.de/static/en/ journals/dgkh/2008-2/dgkh000085.shtml

[51] von Felbert V, Simon D, Braathen LR et al. Behandlung einer linearen Sklerodermie mit wassergefilterter Infrarot-A-Strahlung. Hautarzt 2007; 58: 923-924 PMID 17955204

[52] von Lieven H, Dapper FD. Water-filtered infrared-A radiation: a new technique for hyperthermia of superficial tumors in men. 14th Annual Meeting European Society Hyperthermic Oncology (ESHO). Amsterdam/Niederlande. Abstract Volume, 1994: P9

[53] Winkel R, Hoffmann G, Hoffmann R. Wassergefiltertes Infrarot A (wIRA) hilft Wunden heilen. Der Chirurg 2014; 85: 980-992 doi:10.1007/s00104-014-2809-8 Online publiziert: 12. November 2014. Auch online frei verfügbar http://link.springer.com/article /10.1007/s00104-014-2809-8 http://publikationen.ub.uni-frankfurt. de/frontdoor/index/index/docld/35551 